\title{
Prevalence of benign and malignant tumors in thyroidectomy specimens
}

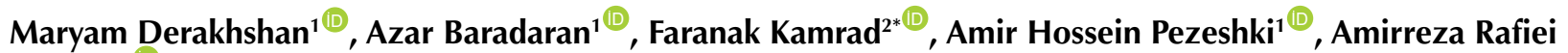 \\ Javazm $^{3 * \mathbb{D}}$
}

${ }^{1}$ Department of Pathology, Isfahan University of Medical Sciences, Isfahan, Iran

${ }^{2}$ Medical Student, Isfahan University of Medical Sciences, Isfahan, Iran

${ }^{3}$ Student Research Committee, Faculty of Nursing and Midwifery, Shahid Sadoughi University of Medical Sciences, Yazd, Iran

\section{Correspondence to:}

Amirreza Rafiei Javazm, Email:

amirrezarafie@yahoo.com

Received: 18 Dec. 2019 Accepted: 23 Apr. 2020 ePublished: 2 Aug. 2020

Keywords: Thyroid Neoplasms; Thyroid Nodule; Prevalence; Epidemiology

\begin{abstract}
Introduction: The most common type of endocrine malignancy is thyroid cancer (TC). TC is the 7 th, 14 th, and 11th most common cancer in women, men, and both sexes, respectively.

Objectives: The aim of this research was to establish the frequency of benign and malignant tumors in thyroid surgery specimens performed in Al-Zahra hospital in Isfahan from 2011 to 2017.

Patients and Methods: This is a retrospective descriptive cross-sectional study. 1345 patients who underwent thyroid surgery were enrolled using census sampling. Data were analyzed using SPSS software (IBM statistics for windows, version 16) using descriptive statistics, mean, standard deviation, frequency, chi-square, and ANOVA. A significance level of less than 0.05 was analyzed.

Results: Of 1345 participants, $237(17.6 \%)$ were males, and $1108(82.4 \%)$ were females. The mean age of benign patients was 46.48, malignant 39.31, and healthy patients for 37.50 years. Since the level of significance is less than 0.05 , there is a significant relationship between the mean age of patients and the frequency of thyroid surgery specimens. The most prevalent benign and malignant thyroid tumors were goiter $(76.8 \%)$ and papillary carcinoma $(92.6 \%)$, respectively.

Conclusion: The results showed that there was a significant relationship between age, gender, and benign and malignant type. Also, the most prevalent benign and malignant thyroid tumors were goiter and papillary, respectively.
\end{abstract}

\section{Introduction}

The most common type of endocrine malignancy is thyroid cancer (TC) (1). TC accounts for $2.1 \%$ of all cancers, according to GLOBOCAN 2012. Its global mortality is also estimated at 0.5 (0.3 in males, 0.6 in females) per 100000 (2). TC is the $7^{\text {th }}, 14^{\text {th }}$, and $11^{\text {th }}$ most common cancer in women, men, and both sexes, respectively (3). Thyroid follicular cells cause two significant groups of TCs; differentiated (papillary and follicular) and undifferentiated (anaplastic, poorly differentiated) carcinomas (4). The etiology of TC is mostly unknown, and so far, only limited risk factors are recognized. Exposure to ionizing radiation, family records, and records of goiter or benign thyroid disorder are among the most straightforward setup risk factors $(5,6)$. Other possible risk factors consist of the use of oral contraceptive pills, extra weight, records of miscarriage, use of fertility drugs, advanced age at pregnancy, and excessive intake of seafood and dietary iodine (7-10).

\section{Key point}

In a retrospective descriptive cross-sectional study on 1345 patients who underwent thyroid surgery, we found the most prevalent benign and malignant thyroid tumors were goiter and papillary carcinoma, respectively.

Radiation, genetic factors, underlying thyroid disease, hormonal factors (more prevalent in women), and nutritional factors, especially iodine, play essential roles in the pathogenesis of TC (11). There are also significant racial and geographic differences in the incidence of TC (12).

The most significant prognostic variables for TC are age and stage (13). TC is twice as conventional in females as in males (14). The 5 -year survival rate for a sample in Tehran was $82.2 \%$ (15).

There is extensive research in Iran on the prevalence and incidence of TC. A study by Kojidi et al, which estimated the prevalence of TC from 1990 to 2010, showed that the 
prevalence of TC has increased in all provinces, especially since 2002, as the year progresses. However, information on subtypes of TC and survival rates has not been provided in this study (16).

Objectives

Currently, there are limited data on the prevalence of TC in Isfahan. The aim of this research was to establish the frequency of benign and malignant tumors in thyroid surgery specimens performed in Al-Zahra hospital in Isfahan from 2011 to 2017.

\section{Patients and Methods}

\section{Study patients}

A retrospective descriptive cross-sectional study was conducted at Isfahan Al-Zahra hospital In November of 2019. The study recruited 1345 users between 2011 and 2017. Sampling is a census sampling method used in this study. Inclusion criteria in this study are all patients admitted to Al-Zahra hospital due to any thyroid mass and underwent surgery. Exclusion criteria included all incomplete pathology reports without a definitive diagnosis. Data were collected from all demographic information, including age, sex, and types of thyroid tumors using patient records.

\section{Ethical issues}

This study was approved by the Isfahan University of Medical Sciences Research Ethics Committee (\#IR.MUI. MED.REC.1398.144), which is in accordance with the principles of the Helsinki Declaration (1964). This study was extracted from M.D thesis of Faranak Kamrad at Isfahan University of Medical Sciences.

\section{Data analysis}

All data were analyzed using SPSS statistical software (IBM Corp. Released in 2013. IBM SPSS Windows Statistics, Version 22.0. Armonk, NY). Descriptive statistics used included mean, standard deviation (SD), and percentage. Also, the chi-square and ANOVA analyses were employed. The level of significance was set to $P<0.05$.

\section{Results}

Of 1345 participants, 237 (17.6\%) were males, and 1108 $(82.4 \%)$ were females. The mean age was $42.91 \pm 13.73$ years. Of the 1345 samples studied, 677 were benign, 662 were malignant, and 6 were normal.

According to Table 1, in 662 cases, 548 (82.8\%) were males, and 114 (17.2\%) were males. In 677 benign reports, $556(82.1 \%)$ were women, and $121(17.9 \%)$ were men. There were 556 benign and 548 malignancies in females.

According to Table 2, the mean age of benign patients was 46.48 , malignant 39.31 , and healthy patients 37.50 years. The mean age of the three groups was compared using ANOVA. Since the significance level is less than 0.05, there is a significant relationship between the mean age of patients and the frequency of thyroid surgery specimens.
Table 1. Trends in the occurrence of thyroid cancer according to age and gender

\begin{tabular}{lccc}
\hline Gender & Age & Benign & Malignant \\
\hline \multirow{4}{*}{ Male } & $0-44$ & $51(42.1 \%)$ & $70(64.1 \%)$ \\
& $45-54$ & $26(21.5 \%)$ & $18(15.8 \%)$ \\
& $55-64$ & $26(21.5 \%)$ & $18(15.8 \%)$ \\
& $65-74$ & $12(9.9 \%)$ & $7(6.1 \%)$ \\
& +75 & $6(5 \%)$ & $1(0.9 \%)$ \\
Female & Total & 121 & 114 \\
& $0-44$ & $301(54.1 \%)$ & $399(72.8 \%)$ \\
& $45-54$ & $105(18.9 \%)$ & $78(14.2 \%)$ \\
& $55-64$ & $107(19.2 \%)$ & $41(7.5 \%)$ \\
& $65-74$ & $40(7.2 \%)$ & $17(3.1 \%)$ \\
& +75 & $3(0.5 \%)$ & $13(2.4 \%)$ \\
\hline
\end{tabular}

Table 2. Descriptive indicators of patients' age by cancer type

\begin{tabular}{lccl}
\hline & Number & Mean & P value* \\
\hline Benign & 677 & $46.48 \pm 12.72$ & \\
\hline Malignant & 662 & $39.31 \pm 13.79$ & \\
Normal & 6 & $37.50 \pm 11.86$ & $<0.001$ \\
Total & 1345 & $42.91 \pm 13.73$ & \\
\hline
\end{tabular}

$* P<0.05$.

Benign TC incidence is the highest in men between $0-44$ years old; this age group includes $42.1 \%$ of cases. The highest prevalence for women is in the $0-44$ age group and 55-64, which comprises $54.1 \%$ and $19.2 \%$ of cases (Table 1).

Malignant TC incidence is the highest in men between $0-44$ years old; this age group includes $42.1 \%$ of cases. The highest rate for women is in the 0-44 age group and 55-64, which comprises $54.1 \%$ and $19.2 \%$ of cases (Table 1 ).

Chi-square test showed that there was a significant relationship between age, gender, and benign and malignant type $(\mathrm{P}<0.001)$.

The most common benign types of TC were goiter (76.8\%), follicular adenoma (9.5\%), and Hashimoto's disease (8.9\%) (Figure 1).

The most common type of malignant thyroid carcinoma was papillary (92.6\%), and the least prevalent was metastatic $(0.3 \%)$ (Figure 2).

\section{Discussion}

The aim of this research was to establish the frequency of benign and malignant tumors in thyroid surgery specimens performed in Al-Zahra hospital in Isfahan from 2011 to 2017.

The findings showed that the most prevalent in benign goiter specimens and papillary malignant specimens were the most frequent. It was also revealed that the age of patients in the three groups of benign, malignant, and healthy was significantly different.

In 2014, a study was conducted by Huan et al. In this 


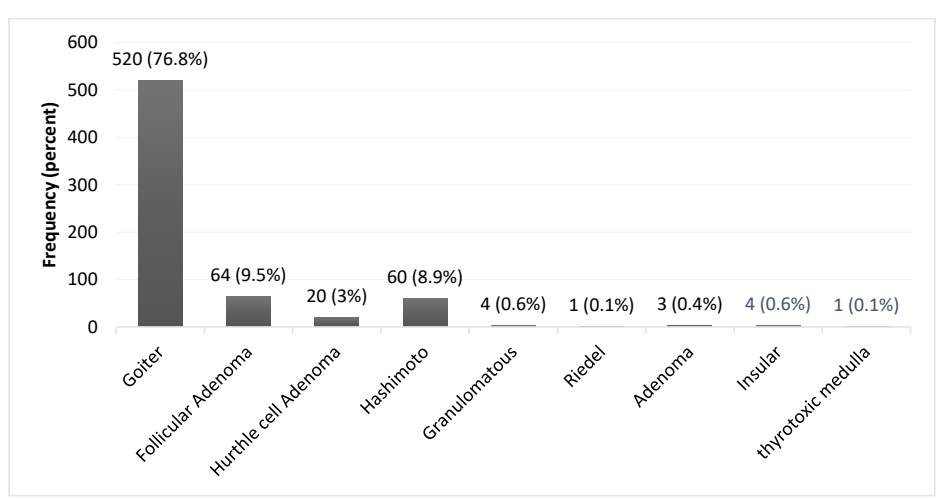

Figure 1. Frequency distribution of benign thyroid cancer types.

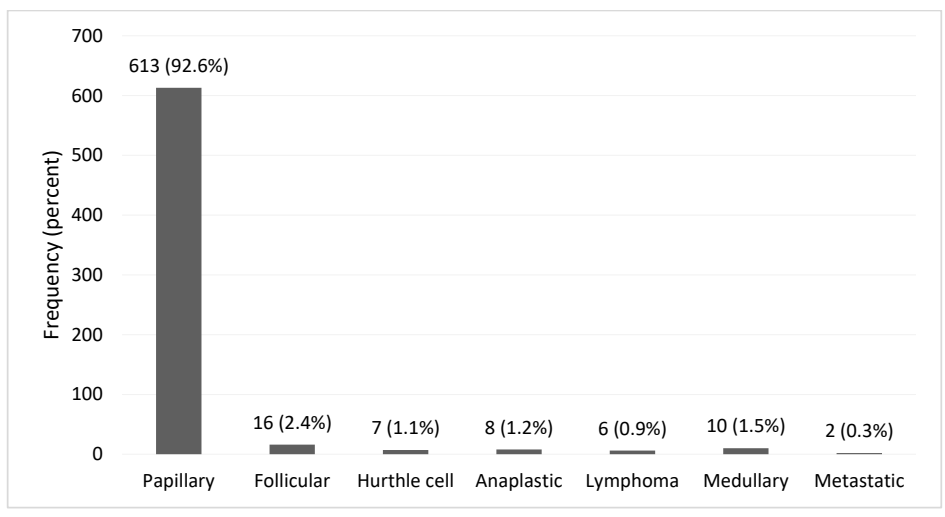

Figure 2. Frequency distribution of malignant types of thyroid cancer.

study, the researchers analyzed the epidemiological and clinical features of thyroid nodules in patients undergoing surgery and identified the risk factors for malignant nodules in order to know more about the differential diagnosis of thyroid nodules. In this study, $65.8 \%$ of the samples were benign (17). However, in the present study, $50.33 \%$ of the samples were benign. Additionally, in the benign nodule group, there were 3227 cases (51.19\%) with nodular goiter, 330 cases $(5.23 \%)$ with Hashimoto's thyroiditis (17). In our study, the most frequent group of benign tumors was goiter and Hashimoto's disease.

A study by Salamanca-Fernández et al, aimed to identify and analyze trends in TC prevalence and mortality in Granada between 1985 and 2013. The results of this study, as in the present study, were the most prevalent among the types of thyroid tumors, papillary type (62.5\% in males and $79.26 \%$ in females) (18).

Additionally, in the study of Sipos et al, papillary was the most prevalent (79\%) among malignant tumors ( $\mathrm{n}=$ 53856 ) in the United States (19).

In 2014, a study was conducted by Huan et al. The average ages of the patients in the malignant nodules group were younger than those in the benign nodules group ( $44.59 \pm 12.22$ versus $49.31 \pm 12.41$ years) (17). The results of this study were consistent with our research.

In our study, the mean age in the benign and malignant groups was 46.48 and 39.31 years, respectively. In the review by Kajidi et al, TC was less prevalent under 14 years of age, with the highest TC incidence in women over 70 years of age, and among men older than 70 , the prevalence of TC was higher (16). In most previous limited studies in Iran, the mean age of both sexes in the age group of 4050 has been reported (20-22). In a survey by Larijani et al (1998-2001), in all provinces of Iran, the highest incidence was in the age group of 45-55 years for women and 5565 years for men, and the impact after age 65 years has increased in both sexes (23). A study by Marjani et al in Golestan province reported the highest incidence of TC in both genders in the age group of 70-79 years (24). In Saudi Arabia, the highest rate of TC is reported in women between the ages of 30 and 39, which is significantly different from the results of the study (25).

Conclusion

The findings showed a significant relationship of age, gender, and benign with malignant type. Also, the most prevalent benign and malignant thyroid tumors were goiter and papillary carcinoma, respectively.

Determine the risk factors of benign and malignant tumors in thyroid surgery specimens and prevent them from exposure. Conducting research aimed at detecting and determining the provincial distribution of this disease 
should be a top priority in the national health arena.

\section{Limitations of the study}

This study was conducted as a single center study, requires further expansion by multi-centric investigations on this subject.

\section{Acknowledgments}

The authors would like to thank the Isfahan University of Medical Sciences, Al-Zahra Hospital, research unit, and all hospital staff for their collaboration.

\section{Authors' contribution}

MD, Supervision of study, the designing protocol of the study, data collection, manuscript writing; FK, Designing protocol of the study, data collection, manuscript writing; ARJ, Designing protocol of the study, data analysis, manuscript writing; $A B$, Designing protocol of the study and data collection, manuscript writing. All authors read and signed the final draft of the manuscript before submission.

\section{Conflicts of interest}

The authors declare no conflicts of interest.

\section{Ethical considerations}

Ethical issues (including plagiarism, data accuracy, duplicate publication) have been ultimately obeyed by the authors.

\section{Funding/Support}

This research received no external funding.

\section{References}

1. Vigneri R, Malandrino P, Vigneri P. The changing epidemiology of thyroid cancer: why is incidence increasing? Curr Opin Oncol. 2015;27:1-7. doi: 10.1097/CCO.0000000000000148.

2. Ferlay J, Soerjomataram I, Dikshit R, Eser S, Mathers C, Rebelo $M$, et al. Cancer incidence and mortality worldwide: sources, methods and major patterns in GLOBOCAN 2012. Int J Cancer. 2015;136:E359-E86. doi: 10.1002/ijc.29210.

3. Akbari M, Abachizadeh K, Khayamzadeh M. Iran cancer report. Qom: Darolfekr. 2008.

4. Fagin JA, Wells Jr SA. Biologic and clinical perspectives on thyroid cancer. N Engl J Med. 2016;375:1054-67. doi: 10.1056/NEJMra1501993.

5. Iribarren C, Haselkorn T, Tekawa IS, Friedman GD. Cohort study of thyroid cancer in a San Francisco Bay area population. Int J Cancer. 2001;93:745-50. doi: 10.1002/ijc.1377.

6. Franceschi S, Preston-Martin S, Dal Maso L, Negri E, La Vecchia C, Mack WJ, et al. A pooled analysis of case-control studies of thyroid cancer. IV. Benign thyroid diseases. Cancer Causes Control. 1999;10:583-95. doi: 10.1023/a:1008907227706.

7. La Vecchia C, Ron E, Franceschi S, Dal Maso L, Mark SD, Chatenoud L, et al. A pooled analysis of case-control studies of thyroid cancer. III. Oral contraceptives, menopausal replacement therapy and other female hormones. Cancer Causes Control. 1999;10:157-66. doi: 10.1023/a:1008832513932.

8. Dal Maso L, La Vecchia C, Franceschi S, Preston-Martin $\mathrm{S}$, Ron E, Levi F, et al. A pooled analysis of thyroid cancer studies. V. Anthropometric factors. Cancer Causes Control. 2000;11(2):137-44. doi: 10.1023/a:1008938520101.

9. Kolonel LN, Hankin JH, Wilkens LR, Fukunaga FH, Hinds MW.
An epidemiologic study of thyroid cancer in Hawaii. Cancer Causes Control. 1990;1:223-34. doi: 10.1007/bf00117474.

10. Negri E, Ron E, Franceschi S, La Vecchia C, Preston-Martin S, Kolonel L, et al. Risk factors for medullary thyroid carcinoma: a pooled analysis. Cancer Causes Control. 2002;13:365-72. doi: 10.1023/a:1015263718760

11. Salabe G. Aetiology of thyroid cancer: an epidemiological overview. Thyroidology. 1994;6:11-9.

12. Parkin D. Cancer incidence in five continents. IARC Sci Publ. 1992;6:960-1.

13. Gulcelik MA, Gulcelik NE, Kuru B, Camlibel M, Alagol H. Prognostic factors determining survival in differentiated thyroid cancer. J Surg Oncol. 2007;96:598-604. doi: 10.1002/ jso. 20845

14. Grubbs EG, Rich TA, Li G, Sturgis EM, Younes MN, Myers $\mathrm{JN}$, et al. Recent advances in thyroid cancer. Curr Probl Surg. 2008;45(3):156-250. doi: 10.1067/j.cpsurg.2007.12.010.

15. Mohagheghi S, Mousavi JS, Tavangar S, Haghpanah V, Lashkari A, Saeidi M, et al. Thyroid cancer in Iran: An epidemiological survey based on cancer data's registered in Tehran. Journal of Medical Council of Iran 2006;23:362-7.

16. Taghavi kojidi H, Farzadfar F, Peykari N, Larijani B, Rahimzadeh S, Rezaei-Darzi E, et al. A comprehensive study on national and subnational trend in thyroid cancer prevalence in the Iranian population, 1990-2010. Iranian Journal of Diabetes and Lipid Disorders. 2016;15(2):91-100. [Persian].

17. Huan Q, Wang K, Lou F, Zhang L, Huang Q, Han Y, et al. Epidemiological characteristics of thyroid nodules and risk factors for malignant nodules: a retrospective study from 6304 surgical cases. Chin Med J (Engl). 2014;127:2286-92.

18. Salamanca-Fernández E, Rodriguez-Barranco $M$, Chang-Chan Y-L, Redondo-Sánchez D, Domínguez-López S, Bayo E, et al. Thyroid cancer epidemiology in South Spain: a populationbased time trend study. Endocrine. 2018;62:423-31. doi: 10.1007/s12020-018-1681-6.

19. Sipos J, Mazzaferri E. Thyroid cancer epidemiology and prognostic variables. Clin Oncol (R Coll Radiol). 2010;22:395404. doi: 10.1016/j.clon.2010.05.004.

20. Larijani B, Aghakhani S, Khajeh-Dini H, Baradar-Jalili R. Clinico-pathological features of thyroid cancer as observed in five referral hospitals in Iran. Acta Oncol. 2003;42:334-7. doi: 10.1080/02841860310001547.

21. Larijani B, Shirzad M, Mohagheghi M, Haghpanah V, MosaviJarrahi A, Tavangar S, et al. Epidemiologic analysis of the Tehran cancer institute data system registry (TCIDSR). Asian Pac J Cancer Prev. 2004;5:36-9.

22. Haghpanah V, Soliemanpour B, Heshmat R, Mosavi-Jarrahi A, Tavangar S, Malekzadeh R, et al. Endocrine cancer in Iran: based on cancer registry system. Indian J Cancer. 2006;43:805. doi: 10.4103/0019-509x.25889.

23. Larijani B, Mohagheghi MA, Bastanhagh MH, MosaviJarrahi AR, Haghpanah V, Tavangar SM, et al. Primary thyroid malignancies in Tehran, Iran. Med Princ Pract. 2005;14:396400.

24. Marjani A, Kabir MJ. Incidence of thyroid cancer in Golestan Province of Iran: Some initial observations. Pak J Med Sci. 2008;24:887-90

25. Hussain F, Iqbal S, Mehmood A, Bazarbashi S, ElHassan T, Chaudhri N. Incidence of thyroid cancer in the Kingdom of Saudi Arabia, 2000-2010. Hematol Oncol Stem Cell Ther. 2013;6:58-64. 